\title{
Telomere position effect is regulated by heterochromatin-associated proteins and NkuA in Aspergillus nidulans
}

\begin{abstract}
Correspondence
Nancy P. Keller

npkeller@wisc.edu
\end{abstract}

Received 1 March 2010

Revised 13 August 2010

Accepted 16 August 2010
Jonathan M. Palmer, ${ }^{1}$ Sandeep Mallaredy, ${ }^{2}$ Dustin W. Perry, ${ }^{2}$ James F. Sanchez, ${ }^{3}$ Jeffrey M. Theisen, ${ }^{4}$ Edyta Szewczyk, ${ }^{5} \dagger$

Berl R. Oakley, ${ }^{5,6}$ Clay C. C. Wang, ${ }^{3}$ Nancy P. Keller ${ }^{4,7}$ and Peter M. Mirabito ${ }^{2}$

\author{
${ }^{1}$ Department of Plant Pathology, University of Wisconsin, Madison, WI 53706, USA \\ ${ }^{2}$ Department of Biology, University of Kentucky, Lexington, KY 40506, USA \\ ${ }^{3}$ School of Pharmacy, University of Southern California, Los Angeles, CA 90089-9023, USA \\ ${ }^{4}$ Department of Medical Microbiology and Immunology, University of Wisconsin, Madison, \\ WI 53706, USA \\ ${ }^{5}$ Department of Molecular Genetics, Ohio State University, 484 West 12th Avenue, Columbus, \\ $\mathrm{OH}$ 43210, USA \\ ${ }^{6}$ Department of Molecular Biosciences, University of Kansas, Lawrence, KS 66045, USA \\ ${ }^{7}$ Department of Bacteriology, University of Wisconsin, Madison, WI 53706, USA
}

\begin{abstract}
Gene-silencing mechanisms are being shown to be associated with an increasing number of fungal developmental processes. Telomere position effect (TPE) is a eukaryotic phenomenon resulting in gene repression in areas immediately adjacent to telomere caps. Here, TPE is shown to regulate expression of transgenes on the left arm of chromosome III and the right arm of chromosome VI in Aspergillus nidulans. Phenotypes found to be associated with transgene repression included reduction in radial growth and the absence of sexual spores; however, these pleiotropic phenotypes were remedied when cultures were grown on media with appropriate supplementation. Simple radial growth and ascosporogenesis assays provided insights into the mechanism of TPE, including a means to determine its extent. These experiments revealed that the KU70 homologue (NkuA) and the heterochromatin-associated proteins HepA, ClrD and HdaA were partially required for transgene silencing. This study indicates that TPE extends at least $30 \mathrm{~kb}$ on chromosome III, suggesting that this phenomenon may be important for gene regulation in subtelomeric regions of $A$. nidulans.
\end{abstract}

\section{INTRODUCTION}

In recent years, research in fungal biology has provided a view that several gene-silencing strategies are important in organismal development. There are the classic studies of silent mating-type loci (HML and HMR) in budding yeast (Laurenson \& Rine, 1992), RNAi-mediated heterochromatin formation in the fission yeast Schizosaccharomyces pombe (Bühler \& Moazed, 2007), and telomere position

tPresent address: Research Center for Infectious Diseases, JosefSchneider-Strasse 2/Bau D15, 97080 Würzburg, Germany.

Abbreviations: AspGD, Aspergillus Genome Database; TPE, telomere position effect; UU, uracil and uridine.

A supplementary table of primers and three supplementary figures are available with the online version of this paper. effect (TPE) in several fungi including Saccharomyces cerevisiae, Candida spp. and Neurospora crassa (Castaño et al., 2005; Gottschling et al., 1990; Rosas-Hernández et al., 2008; Smith et al., 2008). One of the common mechanisms underlying this form of transcriptional silencing has been shown to be chromatin-level control, a process that involves repositioning of nucleosomes and/or post-translational modifications of histone tail residues, which function to alter the availability of DNA to transcriptional machinery (reviewed by Grewal \& Jia, 2007; Jenuwein \& Allis, 2001).

Possibly the best-understood silencing phenomenon is TPE, as it has been demonstrated in several organisms from yeast to humans despite variable chromatin structure among organisms. For example, yeast heterochromatin consists of Sir-protein complexes while in higher eukaryotes 
heterochromatin is composed of histone $\mathrm{H} 3$ methylated at lysine 9 and heterochromatin protein 1 (Schoeftner \& Blasco, 2009). These differing systems of heterochromatic silencing utilize different proteins, yet the underlying similarity is that chromatin is compacted in areas that are transcriptionally silent. Moreover, several of the TPE-regulated genes have important functions for their respective organisms. For example, a subset of the $F L O$ genes of $S$. cerevisiae involved with adhering to surfaces are located near telomeres and thus regulated under this mechanism (Barrales et al., 2008). Additionally, a polygalacturonase gene (PGU1) is located $\sim 25 \mathrm{~kb}$ from a yeast telomere and thus subject to epigenetic regulation (Louw et al., 2010). In Candida glabrata binding to human cells is a key factor for pathogenicity, and adhesion depends on the telomerically located adhesins, which in turn are regulated by telomeric silencing (Castaño et al., 2005). TPE has also been shown to be variable at yeast telomeres, suggesting that not all telomeres have the same silencing capacity (Mondoux \& Zakian, 2007).

Filamentous fungi have also been shown to have chromosomal location effects (Greenstein et al., 2006; Palmer \& Keller, 2010; Robellet et al., 2010); however, telomeric silencing mechanisms have thus far only been studied in $N$. crassa (Smith et al., 2008). Positional effects have been studied genetically through utilization of null mutants in chromatin-associated enzymes, and several of these mutants have been shown to have various pleiotropic effects in $N$. crassa and Aspergillus fumigatus, indicating that primary metabolism may be regulated by this mechanism (Adhvaryu et al., 2005; Freitag et al., 2004; Palmer et al., 2008; Tamaru \& Selker, 2001; Tamaru et al., 2003). Secondary-metabolite gene clusters are also partially regulated by an altered chromatin landscape; specifically a histone deacetylase ( $\mathrm{HdaA})$, the H3K9 methyltransferase ( $\mathrm{ClrD})$ and heterochromatin protein 1 (HepA) are involved in regulation of the sterigmatocystin and penicillin gene clusters (ReyesDominguez et al., 2010; Shwab et al., 2007), while CclA, a Bre2 orthologue involved in histone 3 lysine 4 methylation, is implicated in silencing of the newly identified emodin and F9775A/B gene clusters (Bok et al., 2009).

Here we present evidence for TPE regulation in $A$. nidulans. We show that transgenes located within $20 \mathrm{~kb}$ of the telomere of the left arm of chromosome III in $A$. nidulans are repressed and that silencing extends at least to the SpoC1 cluster boundary $(\sim 30 \mathrm{~kb}$ distal from the telomere). Additionally, we describe TPE at the right arm of chromosome VI near the penicillin gene cluster. NkuA (a KU70 homologue) has a role in TPE, as a null NkuA mutant partially relieves silencing. In addition, proteins involved in heterochromatin maintenance are involved in TPE, as partial derepression was observed in null mutant backgrounds of HepA, ClrD and HdaA.

\section{METHODS}

General. Fungal strains used in this study are listed in Table 1; all primers used are listed in Supplementary Table S1, available with the online version of this paper. All strains were maintained on glucose minimal medium (GMM) (Shimizu \& Keller, 2001) at $37^{\circ} \mathrm{C}$ and when appropriate were supplemented with $1.2 \mathrm{~g}$ uracil $\mathrm{l}^{-1}, 1.2 \mathrm{~g}$ uridine $1^{-1}, 0.5 \mu \mathrm{M}$ pyridoxine. $\mathrm{HCl}, 2.5 \mu \mathrm{M}$ riboflavin. $\mathrm{HCl}, 100 \mu \mathrm{M}$ arginine and $1 \mu \mathrm{M} p$-aminobenzoate. In silico analysis was done using the Aspergillus Genome Database, AspGD (http://www. aspergillusgenome.org), and all locus identification numbers correspond to the current annotation of AspGD (Arnaud et al., 2010).

Creation of fungal strains. Gene replacement mutants were generated by transformation of suitable recipient strains with gene replacement cassettes constructed using fusion PCR (Szewczyk et al., 2006; Yang et al., 2004). Transformation was done essentially as in Miller et al. (1985), with the exception of embedding protoplasts in top agar $(0.75 \%)$. For example, AN5091 was disrupted with a PCR product consisting of a $0.88 \mathrm{~kb}$ upstream flanking region, a $0.92 \mathrm{~kb}$ downstream flanking region, and a $1.97 \mathrm{~kb}$ PCR fragment of the $A$. fumigatus pyrG gene as a marker gene to create TJMP6.9. All original mutants were created essentially in the same fashion according to the following: TDP1-1, 1-2, 2-7 and 2-12 ( $\triangle$ AN5092:: AfpyrG), TSM18-3 ( $\Delta$ AN5092:: GrypfA), TMM11 ( $\triangle$ AN5495:: AfpyrG), TSM11-3, 11-4


were created in a TN02A7 background, and TJMP6.9 ( $\triangle$ AN5091:: AfpyrG) was created in the RJMP1.49 background. Replacement of the target sequence with the selectable marker was confirmed by Southern blot analysis and by PCR using primers lying outside the gene replacement cassettes. Prototrophic strains were constructed by independently crossing TJMP6.9 and TDP1-1 to RJMP101.5 to construct RJMP115.3 and RJMP116.3. TJMP16.1 was constructed by transformation of TJMP6.9 with pJW53 harbouring the A. nidulans pyroA gene (Tsitsigiannis et al., 2004). Crossing RJMP1.35 with SMC73.1-105 created RJMP121.7 and RJMP121.4, while RJMP122.6, RJMP123.3, RJMP125.20 and RJMP127.4 were created by independently crossing RJMP1.59 with SMC72-1, SMC73.1-5, SMC58-7 and SMC79-13 respectively. Finally, crosses between TJMP50.3 and SMC58-7 yielded RJMP135.11, and TJMP50.3 and SMC79-13 yielded RJMP131.7.

Semi-quantitative reverse transcriptase PCR. Equal numbers of conidia from all strains were inoculated in YSC medium (Lies et al., 1998) containing uracil and uridine (UU) and incubated at $30{ }^{\circ} \mathrm{C}$ overnight. Cells were harvested, frozen in liquid nitrogen, and total RNA was isolated using the procedure of Timberlake (1980). Ten micrograms of total RNA was used to make single-stranded cDNA using the Superscript III kit and the protocol recommended by the manufacturer (Invitrogen). Serial 1:10 dilutions of single-stranded cDNA were used as template in PCRs, and equal volumes of the PCRs were separated on a $1 \%(\mathrm{w} / \mathrm{v})$ agarose gel. The gel was stained with ethidium bromide and imaged using a Typhoon 8600 scanner. The intensity of the bands was quantified using ImageQuant software. The band intensity of each AfpyrG PCR product was divided by the actin band intensity from the same dilution to give an expression ratio AfpyrG/actin for each sample. The AfpyrG/actin ratios from the $0.1 \times$ cDNA reactions for each TDP sample were divided by the ratio from the TMM11 control to give a relative expression level for each sample.

Physiology experiments. Prototrophic strains in a wild-type veA $\left(v e A^{+}\right)$genetic background were used for radial growth measurements and quantification of ascospores. Radial growth assays were conducted at $37^{\circ} \mathrm{C}$ by measuring colony diameter after 3 days on plates of solid medium [GMM and GMM with appropriate supplementation (uridine and uracil, UU; pyridoxine, $\mathrm{P}$ )] that were centrally point-inoculated with $\sim 2000$ spores. Quantification of ascospores was done on overlayinoculated cultures that were set up by pipetting $1 \times 10^{6}$ conidia into $0.75 \%$ molten agar that was subsequently poured over $1.5 \%$ solid agar in Petri dishes. Cultures were incubated at $37{ }^{\circ} \mathrm{C}$ in the dark for 57 days and agar cores were taken from the plates with a $1.2 \mathrm{~cm}$ cork 
Table 1. Strains used in this study

\begin{tabular}{|c|c|c|}
\hline Strain & Genotype & Source \\
\hline TN02A7 & pyrG89 pyroA4 riboB2 $\triangle n k u A:: \operatorname{argB}$ veA1 & Nayak et al. (2006) \\
\hline TDP1-1 & pyrG89 pyroA4 riboB2 $\triangle \mathrm{AN} 5092::$ AfpyrG $\triangle n k u A:: \operatorname{argB} v e A 1$ & This study \\
\hline TDP1-2 & pyrG89 pyroA4 riboB2 $\triangle \mathrm{AN} 5092::$ AfpyrG $\triangle n k u A:: \operatorname{argB}$ veA1 & This study \\
\hline TDP2-7 & pyrG89 pyroA4 riboB2 $\triangle \mathrm{AN} 5092::$ AfpyrG $\triangle n k u A:: \operatorname{argB}$ veA1 & This study \\
\hline TDP2-12 & pyrG89 pyroA4 riboB2 $\triangle \mathrm{AN} 5092:$ : AfpyrG $\triangle n k u A:: \operatorname{argB} v e A 1$ & This study \\
\hline TMM11 & pyrG89 pyroA4 riboB2 $\triangle \mathrm{AN} 5495:$ : AfpyrG $\triangle n k u A:: \operatorname{argB}$ veA1 & This study \\
\hline TSM18-3 & pyrG89 pyroA4 riboB2 $\triangle \mathrm{AN} 5092::$ GrypfA $\triangle n k u A:: \operatorname{argB}$ veA1 & This study \\
\hline TSM11-3 & pyrG89 pyroA4 riboB2 $\triangle \mathrm{AN} 5092::$ AfpyroA $\triangle n k u A:: \operatorname{argB}$ veA1 & This study \\
\hline TSM11-4 & pyrG89 pyroA4 riboB2 $\triangle \mathrm{AN} 5092::$ AfpyroA $\triangle n k u A:: \operatorname{argB}$ veA1 & This study \\
\hline TSM11-10 & pyrG89 pyroA4 riboB2 $\triangle \mathrm{AN} 5092::$ AfpyroA $\triangle n k u A:: \operatorname{argB} v e A 1$ & This study \\
\hline TSM3-1 & pyrG89 pyroA4 riboB2 $\triangle \mathrm{AN} 4432::$ AfpyroA $\triangle n k u A:: \operatorname{argB}$ veA1 & This study \\
\hline RJMP1.49 & pyrG89 pyroA4 $\triangle n k u A:: \operatorname{argB}$ & Shaaban et al. (2010) \\
\hline TJMP6.9 & pyrG89 pyroA4 $\triangle \mathrm{AN} 5091::$ AfpyrG $\triangle n k u A:: \operatorname{argB}$ & This study \\
\hline RJMP101.5 & pyrG89 wA3 & This study \\
\hline RJMP115.3 & pyrG89 $\triangle \mathrm{AN} 5091:$ : AfpyrG & This study \\
\hline RJMP116.3 & pyrG89 $\triangle \mathrm{AN} 5092:$ : AfpyrG & This study \\
\hline TJMP45.2 & $\Delta n k u A:: \arg B$ & Shaaban et al. (2010) \\
\hline TJMP16.1 & pyrG89 $\triangle \mathrm{AN} 5091::$ AfpyrG $\Delta n k u A:: \arg B$ & This study \\
\hline RJMP1.19 & pyroA4 $\triangle n k u A:: \arg B$ & Shaaban et al. (2010) \\
\hline TMSII2.4 & pyroA4 pyrG89 $\triangle \mathrm{PbII}::$ AppyrG $\triangle n k u A:: \operatorname{argB}$ & Shaaban et al. (2010) \\
\hline TMS8.2 & pyroA4 pyrG89 Ap pyrG $\triangle n k u A:: \operatorname{argB}$ & M. Shaaban \& N. P. Keller, unpublished \\
\hline SMC58-7 & $\triangle \mathrm{AN} 5092:$ : AfpyroA pyroA4 $\triangle c l r D:$ : AfpyrG pyrG89 $\operatorname{argB2}$ yA2 veA1 & This study \\
\hline SMC73.1-5 & riboB2 $\triangle \mathrm{AN} 5092:$ : AfpyroA pyroA4 $\Delta$ hdaA:: AfpyrG pyrG89 veA1 & This study \\
\hline SMC72-1 & pyrG89 $\triangle$ hepA:: AfpyrG $\triangle \mathrm{AN} 5092:$ : Afpyro pyroA4 riboB2 wA2 yA2 veA1 & This study \\
\hline SMC73.1-105 & $\triangle \mathrm{AN} 5092:$ : AfpyroA pyroA4 pyrG89 argB2 riboB2 wA2 veA1 & This study \\
\hline SMC79-13 & pyrG89 $\Delta$ hstA::argB $\triangle \mathrm{AN} 5092::$ Afpyro pyroA4 riboB2 pabaA1 yA2 veA1 & This study \\
\hline RJMP1.59 & pyrG89 pyroA4 & This study \\
\hline TJMP50.3 & pyrG89 & This study \\
\hline RJMP1.35 & pyroA4 trpC801 & This study \\
\hline RDIT9.32 & Wild-type & Tsitsigiannis et al. (2004) \\
\hline RJMP121.7 & pyroA4 $\triangle \mathrm{AN} 5092:$ : AfpyroA & This study \\
\hline RJMP121.4 & pyroA4 $\triangle \mathrm{AN} 5092:$ : AfpyroA veA1 & This study \\
\hline RJMP122.6 & pyroA4 $\triangle \mathrm{AN} 5092::$ AfpyroA $\Delta$ hepA:: AfpyrG & This study \\
\hline RJW110.4 & $\Delta h e p A:: \operatorname{Af} p y r G$ & Reyes-Dominguez et al. (2010) \\
\hline RJMP123.3 & pyroA4 $\triangle$ AN5092:: AfpyroA $\Delta h d a A::$ AfpyrG & This study \\
\hline RMS1.22 & $\Delta h d a A::$ AfpyrG & Shaaban et al. (2010) \\
\hline RJMP125.20 & pyroA4 $\triangle$ AN5092:: AfpyroA $\Delta c l r D:$ : AfpyrG & This study \\
\hline RJMP135.11 & $\Delta c l r D::$ AfpyrG & This study \\
\hline RJMP127.4 & pyroA4 $\triangle \mathrm{AN} 5092:$ : AfpyroA $\Delta$ hstA::argB & This study \\
\hline RJMP131.7 & $\Delta h s t A:: \arg B$ & This study \\
\hline
\end{tabular}

borer. This method of inoculation allows cleistothecia to develop evenly across the Petri dish. Ascospores were quantified using a haemocytometer and represented as ascospores per square millimetre. All experiments were completed at least in triplicate and statistical analysis was done using Prism 5 software.

\section{RESULTS}

\section{Discovery of TPE at the left arm of chromosome III}

We were originally interested in AN5092 because of its putative function assigned by the genome annotation. AN5092 is predicted to encode a full-length RecQ protein, which is an orthologue of the telomere-linked helicase (TLH) gene family described in Magnaporthe oryzae
(Rehmeyer et al., 2009). TLH genes are found in close proximity to telomeres of some species of filamentous fungi and are similar in sequence to telomerically located $\mathrm{Y}^{\prime}$ elements in S. cerevisiae (Rehmeyer et al., 2006). Analysis of TLH-related DNA sequences in A. nidulans revealed that they were present near the ends of six chromosomes (Clutterbuck \& Farman, 2008); however, only AN5092 appears to be full-length according to the criteria of Rehmeyer et al. (2009).

To investigate the role of AN5092, we replaced the ORF with the A. fumigatus pyrG gene (AfpyrG), which complements the uracil/uridine auxotrophy conferred by pyrG89. A $\triangle n k u A$ genetic background (TN02A7) was used to increase the likelihood of obtaining the desired mutant (Nayak et al., 
2006). Four of thirteen transformants screened by Southern blot and PCR analyses had simple gene replacements (AN5092::AfpyrG). All four strains (TDP1-1, 1-2, 2-7 and 2-12) produced smaller colonies on minimal medium (GMM) than control transformants, where AfpyrG was used to replace the nonessential, non-telomerically located gene AN5495 (Fig. 1a). We hypothesized that partial repression of the AfpyrG gene could be responsible for this phenotype. Consistent with this idea, the colony size defect of all four AN5092:: AfpyrG strains was completely relieved by supplementing the media with uracil and uridine (UU) (Fig. 1a). Moreover, we have used AfpyrG for gene replacements at several other locations in the genome without observing a growth defect (data not shown). We therefore attributed the growth defect of the TDP strains to partial uracil auxotrophy, not the loss of the ORF corresponding to AN5092.

The partial uracil auxotrophy of the TDP strains suggested that expression of AfpyrG is reduced in these strains compared to controls. To test this, we analysed AfpyrG mRNA levels in our strains by semiquantitative RT-PCR. Fig. 1(b) shows that expression of the AfpyrG transgene is reduced by approximately one-half in TDP strains compared to a control strain. These results indicated that the reduced colony growth rate of TDP strains is likely due to reduced levels of AfpyrG expression.

\section{Repression is independent of transgene or transgene orientation}

To determine whether the orientation of the transgene at AN5092 is required for the reduced expression described above, we repeated the deletion of AN5092 using a construct in which the direction of AfpyrG transcription was opposite to that in the AN5092::AfpyrG strains (schematically drawn in Fig. 2a). A transformant (TSM183) was obtained and confirmed by PCR and Southern analysis (Fig. 2b). Like TDP1-1, the AN5092:: GrypfA strain produced small colonies on minimal medium and normal-sized colonies on medium containing UU (data not shown). Thus, a specific orientation of the transgene inserted near the telomere of chromosome III was not required for silencing.

Additionally, we reasoned that the growth defect could be specific to uracil metabolism; thus, we repeated the deletion of AN5092 using an alternative selectable marker, A. fumigatus pyroA (AfpyroA). Three strains (TSM11-3, 114 and 11-10) were isolated and confirmed by PCR and Southern analysis (Fig. 2b). The nonessential gene AN4432 (centrally located in chromosome III) was disrupted with AfpyroA and served as a control strain (data not shown). All three strains (TSM11-3, 11-4 and 11-10) produced small colonies on medium lacking pyridoxine and the growth defect was remedied by addition of pyridoxine to the medium, whereas the control strain produced normalsized colonies on both media (Supplementary Fig. S1).

\section{In silico analysis of the telomere-proximal left arm of chromosome III}

To gain potential insight into the silencing of the transgene located at AN5092, we employed an in silico approach to determine the chromosomal context of AN5092. Using the AspGD (http://www.aspergillusgenome.org), we determined (a)



(b)

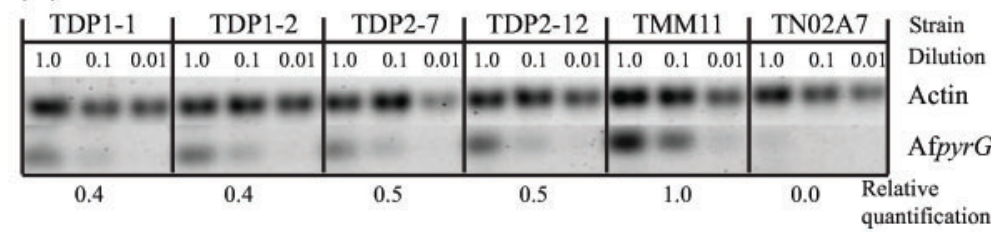

Fig. 1. (a) Gene replacement of AN5092 with the $A$. fumigatus pyrG gene resulted in a strain with visible growth defects on minimal medium compared to a control strain (TMM11). The observed phenotype was remedied when cultures were grown on medium containing the appropriate supplements, in this case uridine and uracil. (b) The growth reduction on minimal medium correlates with repression of the AfpyrG transgene located at AN5092. The control strain (TMM11) contains AfpyrG integrated at the nonessential gene AN5495. Semiquantitative RT-PCR revealed that relative AfpyrG expression levels were 0.4 for TDP1-1 and TDP1-2 and 0.5 for TDP2-7 and TDP2-12. 


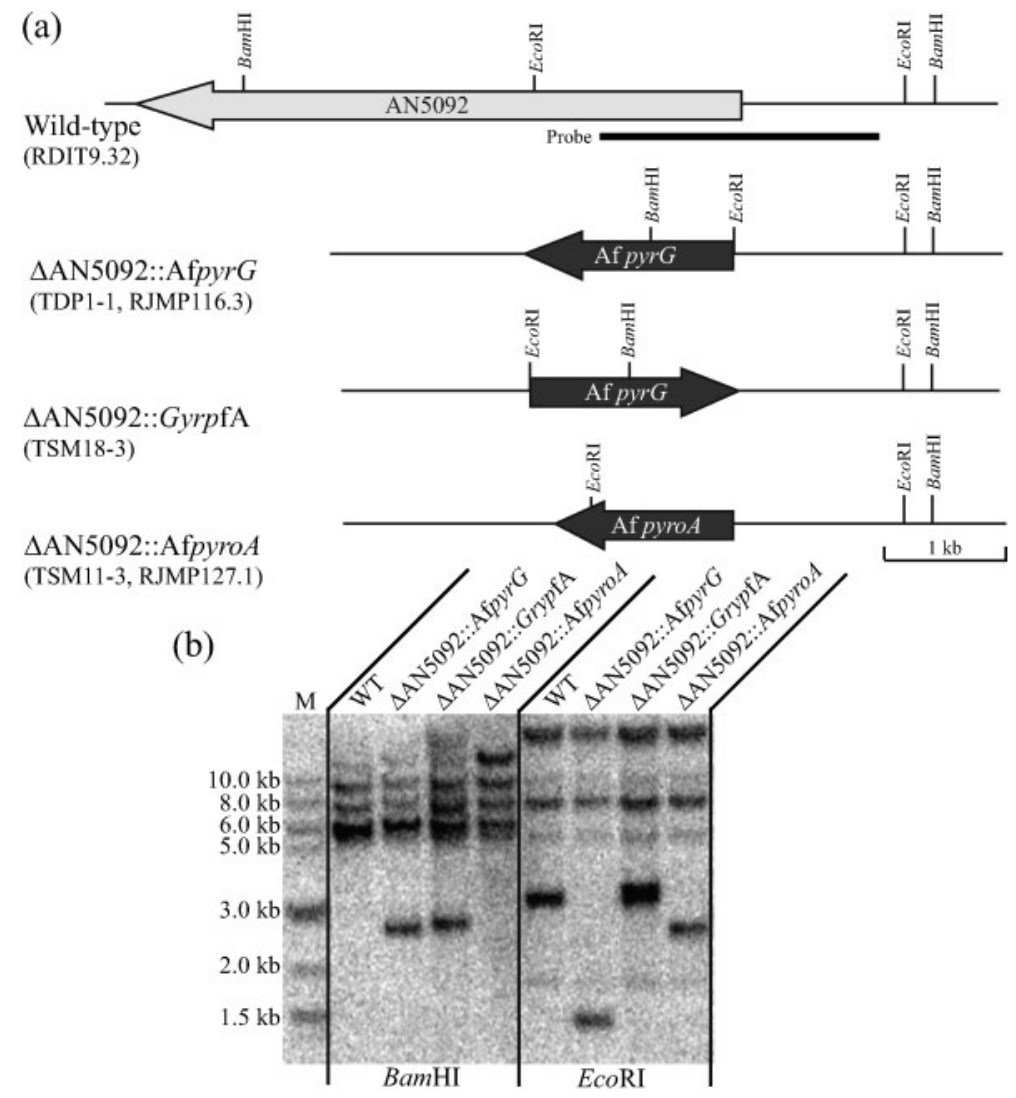

Fig. 2. Schematic of gene disruption strategies (a) and Southern analysis of mutants constructed at the AN5092 locus (b). The AN5092 locus was replaced with three constructs: A. fumigatus pyrG, $A$. fumigatus pyrG in the opposite orientation (GrypfA), and the $A$. fumigatus pyro $A$ gene. Due to the repetitive nature of TLH-like sequences, Southern blots produced a considerable amount of background. Strains used for Southern blots were: WT, RDIT9.32; $\triangle$ AN5092::AfpyrG, RJMP116.3; $\triangle$ AN5092:: GrypfA, TSM18-3;

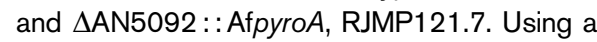
radiolabelled probe corresponding to a portion of the AN5092 locus and the 5' flanking region, in a BamHI digestion the following bands were

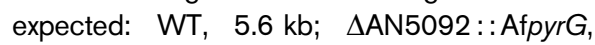
$2.6 \mathrm{~kb} ; \quad \triangle \mathrm{AN} 5092:$ :GrypfA, $2.8 \mathrm{~kb} ; \quad$ and $\triangle$ AN5092::AfpyroA, >10 kb. An EcoRI diges-


$1.4 \mathrm{~kb} ; \quad \triangle \mathrm{AN5092::GrypfA}, \quad 3.2 \mathrm{~kb} ; \quad$ and

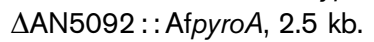

that AN5092 is located $\sim 2 \mathrm{~kb}$ from the end of chromosome III. According to Clutterbuck \& Farman (2008), there is a sequence gap of $18.4 \mathrm{~kb}$ between the telomere cap and the genome annotation. Taken together, these analyses place AN5092 approximately $20 \mathrm{~kb}$ from the telomere of chromosome III (Fig. 3). Further analysis revealed the presence of the SpoC1 cluster telomere distal to AN5092. The SpoC1 cluster, described by Gwynne et al. (1984), is a $38 \mathrm{~kb}$ cluster of developmentally regulated transcripts, which are flanked by two $1.1 \mathrm{~kb}$ repetitive sequences (RPT3). While two of the SpoC1 cluster genes are annotated in the AspGD genome sequence, the remaining genes are absent. Our in silico analysis indicates that the RPT3 flanking sequences of the SpoC1 cluster are located near the current annotation of AN5091 and AN5081. Expanding on these data, analysis of the orientation of genes presented by Gwynne et al. (1984) matches perfectly with the annotation found in the AspGD. Therefore we conclude that the SpoC1 cluster corresponds to a $38 \mathrm{~kb}$ stretch located on chromosome III and the boundary is located $30 \mathrm{~kb}$ from the telomere (Fig. 3). In addition to AN5092 there are two putative ORFS located between the SpoC1 cluster and the end of chromosome III. AN5093 is likely not a functional gene as it is composed of repetitive DNA sequence, and AN5091 encodes a putative methyltransferase with some similarity to LaeA, a protein involved in regulation of secondary metabolite gene clusters (Bok \& Keller, 2004).

\section{Repression extends at least to the boundary of the SpoC1 cluster}

Via Northern analysis, we were unable to detect expression during a developmental time-course in a wild-type strain (RDIT9.32) of the putative telomere-distal gene AN5091, which is more than $30 \mathrm{~kb}$ from the telomere (Fig. 3, Supplementary Fig. S2). This indicated that silencing might extend beyond the AN5092 locus. In a similar fashion to disruption of AN5092, the AN5091 locus was replaced with the AfpyrG gene and the replacement confirmed by Southern analysis (Fig. 4). The resulting phenotype was nearly identical to that of the AN5092:: AfpyrG mutant, with transformants requiring UU supplementation for normal growth and ascosporogenesis (Fig. 5a, b). Taken together, our data suggest that TPE functions regardless of the transgene or orientation, and extends at least to the SpoC1 cluster boundary.

\section{TPE can be assayed via radial growth and ascospore production}

In order to study TPE in prototrophic strains and a wildtype background, we attempted to outcross TDP1-1 (AN5092::AfpyrG). Heterokaryons were formed and selected on minimal medium, and cleistothecia were allowed to form. All cleistothecia examined from the attempted outcross were devoid of ascospores. Outcrosses of the other three AN5092:: AfpyrG mutants gave the same 


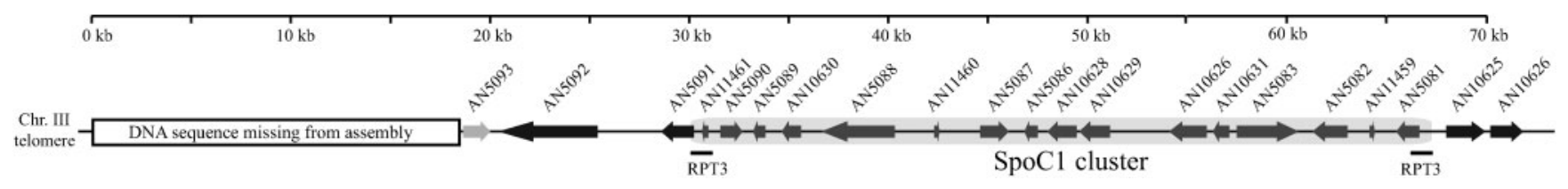

Fig. 3. Scale schematic representation of the left telomere of chromosome III of $A$. nidulans. AN5092 is located $\sim 20 \mathrm{~kb}$ from the telomere of the left arm of chromosome III. Clutterbuck \& Farman (2008) describe that $18.4 \mathrm{~kb}$ of DNA is missing between the telomere of chromosome III and the genome assembly. Bioinformatic analysis places the SpoC1 cluster approximately $30 \mathrm{~kb}$ from the telomere of chromosome III, spanning the current annotation from AN5091 to AN5081. Three putative open reading frames exist between the SpoC1 cluster and the end of the current annotation in the AspGD: AN5093 is unlikely to be a functional gene as it contains repetitive sequences, AN5092 is a putative TLH-like gene, and AN5091 encodes a methyltransferase with sequence homology to LaeA.

result. Since TDP1-1 required UU for normal vegetative growth and the transgene located at AN5092 was the only functional $\operatorname{pyr} G$ gene in the cross, we hypothesized that repression of AfpyrG was responsible for the ascosporogenesis defect. Indeed, supplementing the medium with UU remedied the ascosporogenesis defect. Analogous results were obtained with crosses involving TSM11 strains, in which AfpyroA at AN5092 was the only functional pyroA gene. These crosses produced ascospores only when pyridoxine was included in the medium. Thus, the limited expression of the transgene remedying a nutritional deficiency prevents ascosporogenesis.

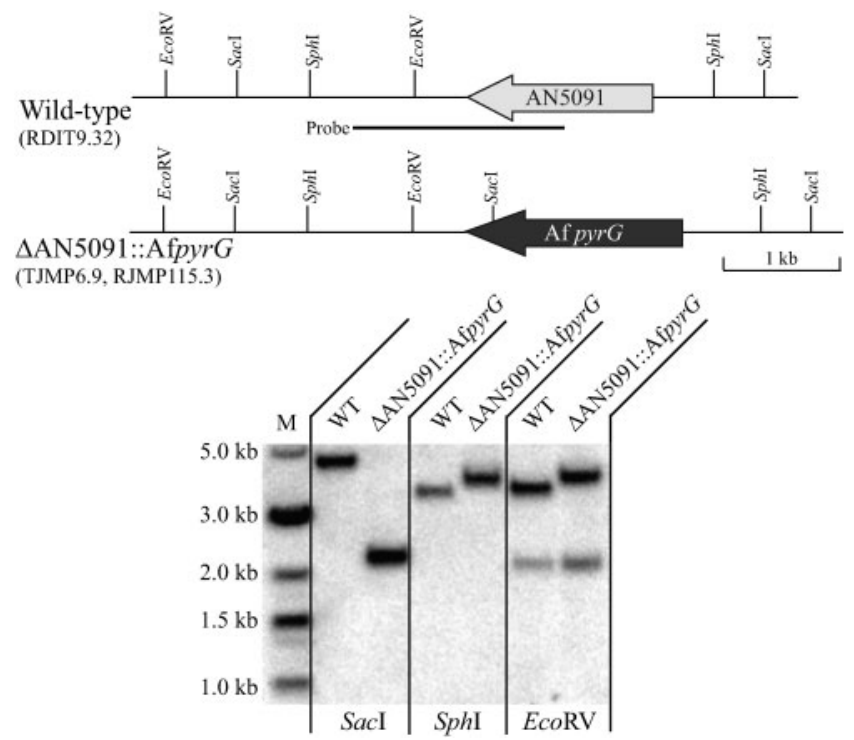

Fig. 4. The AN5091 locus was replaced with the A. fumigatus pyrG gene as drawn schematically. Strains were confirmed by Southern analysis. Strains used were WT (RDIT9.32) and $\triangle A N 5091::$ AfpyrG (RJMP115.3). A Sacl digestion predicted

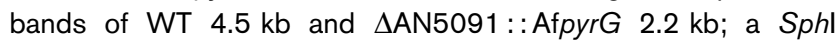

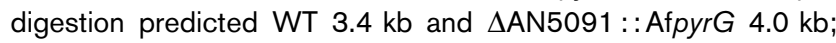
while an EcoRV digestion predicted WT $3.4 \mathrm{~kb}+2.1 \mathrm{~kb}$ and

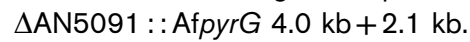

(a)

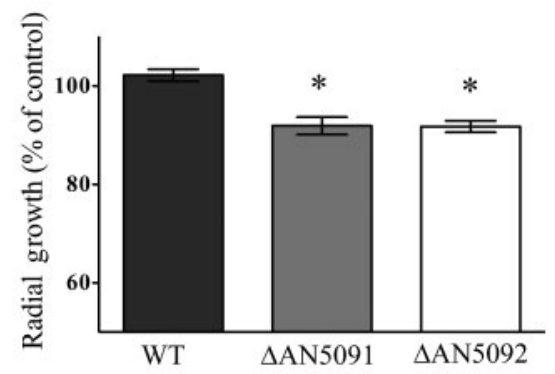

(b)



Fig. 5. (a) Prototrophic strains exhibiting TPE display a quantifiable growth defect on minimal medium versus minimal medium with supplements. Integration of AfpyrG at either AN5092 or AN5091 results in the same phenotype, suggesting that TPE extends at least $30 \mathrm{~kb}$ from the telomere. WT, RDIT9.32; $\triangle$ AN5091, RJMP115.3; $\triangle$ AN5092, RJMP116.3. (b) Sexual development is aberrant in TPE strains. Macroscopic cleistothecial development is unaffected by integration of AfpyrG at either the AN5092 or the AN5091 locus; however, ascospore production is severely debilitated. Quantification of ascosporogenesis indicates that TPE strains produce three orders of magnitude fewer ascospores when grown on minimal medium compared to medium with appropriate supplements. WT, RDIT9.32; $\triangle$ AN5091, RJMP115.3; and $\triangle$ AN5092, RJMP116.3. Note that the $y$-axis scale is logarithmic. Means \pm SD are plotted; asterisks indicate statistically significant differences between wild-type and other strains $(P<0.001$ using Student's $t$-test). 
Most laboratory strains of $A$. nidulans harbour the veA1 allele, which makes them essentially blind to light so they produce asexual conidia regardless of whether grown in the light or dark, with limited sexual development. Therefore, to further examine TPE in the context of sexual development we used wild-type $\left(v e A^{+}\right)$strains. TPE repression of AfpyrG at the AN5092 locus (RJMP116.3) reduced production of ascospores by approximately three orders of magnitude on minimal medium compared to medium supplemented with UU (GMM=618 \pm 405 ascospores $\mathrm{mm}^{-2} ; \quad \mathrm{GMM}+\mathrm{UU}=68083 \pm 8527$ ascospores $\mathrm{mm}^{-2}$ ) (Fig. 5b). In order to confirm that silencing of transgenes was solely responsible for the observed pleiotropic phenotypes, we grew a pyroA4 mutant (RJMP1.59) on medium with decreasing concentrations of pyridoxine and were able to replicate the reduction in radial growth and loss of ascospore production observed in the AN5092::AfpyroA strain (Supplementary Fig. S3).

\section{TPE exists at the right arm of chromosome VI}

A recent study aimed at assessing the role of repetitive sequences flanking the penicillin gene cluster resulted in creation of a mutant with a transgene integrated in close proximity to the telomere of chromosome VI (Shaaban et al., 2010). In this mutant ( $\Delta$ PbII, TMSII2.4), a $30 \mathrm{~kb}$ piece of DNA $\sim 8.5 \mathrm{~kb}$ from the telomere was replaced with the A. parasiticus pyrG gene (Shaaban et al., 2010). Thus this strain allowed us to assay putative TPE silencing effects at chromosome VI. Similar to results from analysis of transgene repression on chromosome III, a reduction in radial growth and ascospore production was quantifiable and was partially remedied by appropriate supplementation of the medium (Fig. 6a, b). A growth defect remains in this strain with UU supplementation, hypothesized to be due to loss of uncharacterized ORFs (Shaaban et al., 2010).

\section{Heterochromatin-associated proteins and NkuA are involved in TPE}

Several proteins have been shown to be required for TPE in other organisms, including KU70/80 (NkuA/B) proteins (Boulton \& Jackson, 1998; Mishra \& Shore, 1999; RosasHernández et al., 2008), as well as heterochromatin protein 1 , the lysine 9 histone 3 methyltransferase, and histone deacetylases (Ottaviani et al., 2008). By utilizing radial growth and ascosporogenesis assays we were able to elucidate the involvement of several heterochromatinassociated proteins (HepA, ClrD, HdaA and HstA) in TPE at chromosome III by creating double mutants of AN5092:: AfpyroA and null mutants of HepA, ClrD, HdaA or HstA. Fig. 7 shows that both radial growth and ascosporogensis were identical to wild-type in the single chromatin mutants but were partially derepressed in HepA, ClrD and HdaA AN5092:: AfpyroA double mutants, thus implicating all three of these proteins as players in TPE regulation. HdaA, a histone deacetylase, had the strongest derepressive effect. However the $\Delta$ hstA AN5092::AfpyroA
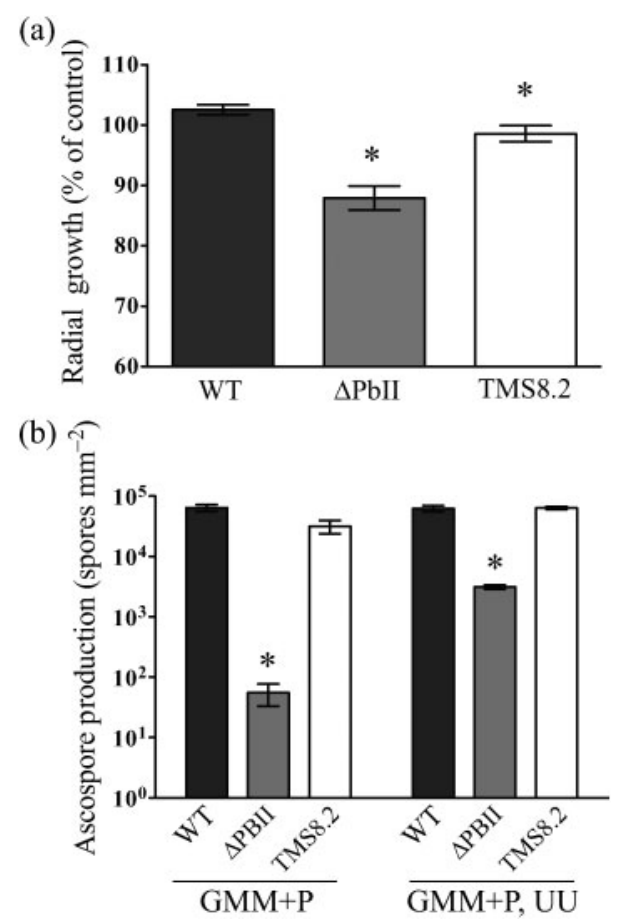

Fig. 6. Physiological experiments with mutants created by Shaaban et al. (2010) allowed for determination of TPE at chromosome VI. (a) A radial growth assay illustrates the quantifiable difference in growth of $\triangle \mathrm{Pbll}$ ( $A$. parasiticus pyrG integrated $\sim 8.5 \mathrm{~kb}$ from the telomere of chromosome VI-R) when grown on GMM versus $\mathrm{GMM}+\mathrm{UU}$. The isogenic control strain (TMS8.2) contains the $A$. parasiticus pyrG gene located ectopically and also shows a slight reduction in growth on GMM versus GMM + UU compared to WT (RJMP1.19); however, radial growth of $\Delta \mathrm{Pbll}$ is significantly reduced compared to TMS8.2. (b) Ascosporogenesis assays mirror the radial growth assay, where $\triangle$ Pbll produces significantly fewer ascospores than either the WT or TMS8.2. These data illustrate TPE repression of the $A$. parasiticus pyrG transgene when located near the telomere of chromosome VI. Means \pm SD are plotted; asterisks indicate statistically significant differences between wild-type and other strains at $P<0.001$ using Student's $t$-test.

double mutant did not show increased radial growth or ascospore production compared to the control strain (Fig. 7a, b, c).

Using a similar approach to test the involvement of NkuA we created a double AN5091::AfpyrG $\Delta n k u A$ mutant. Consistently, this mutant produced more ascospores than the single AN5091::AfpyrG mutant by one order of magnitude (Fig. $7 \mathrm{~d}$ ). Together, these data suggest that NkuA, HepA, ClrD, HstA and HdaA are not involved in radial growth or ascospore production under the conditions tested; however, in $\Delta n k u A, \Delta h e p A, \Delta c l r D$ and $\Delta h d a A$ genetic backgrounds there is partial derepression of the telomerically located transgenes. None of the double mutants were able to restore growth or ascospore production 
(a)

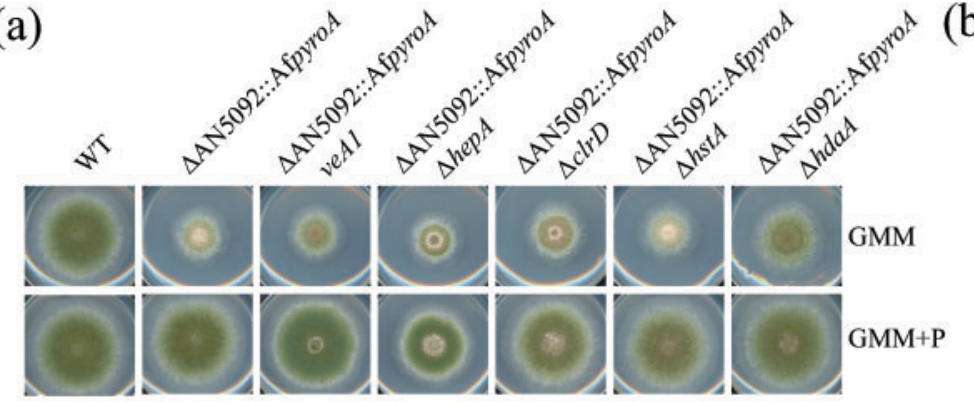

(c)

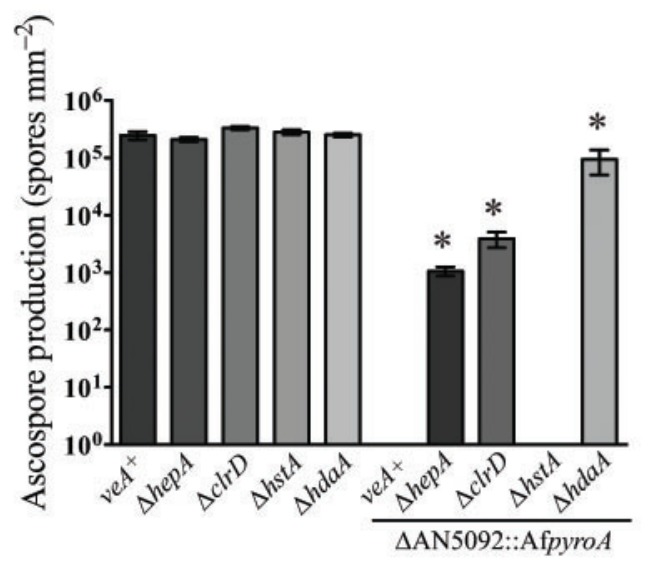

(b)

(d)
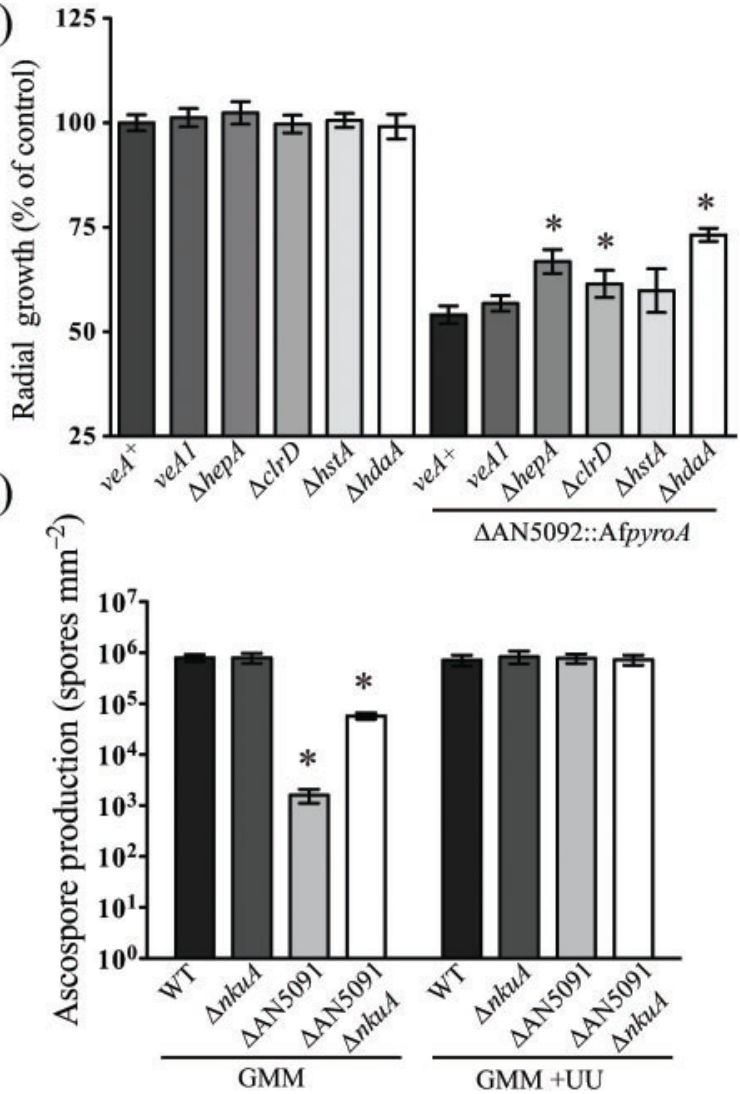

Fig. 7. In order to address the mechanism of TPE in A. nidulans, radial growth and ascosporogenesis assays were conducted on double mutants. (a) Radial growth assays illustrate the reduction in growth on GMM versus $G M M+$ pyridoxine $(P)$ of strains harbouring $\triangle \mathrm{AN} 5092:$ : AfpyroA. (b) Quantification of the radial growth assay indicates that $\mathrm{HepA}, \mathrm{ClrD}$ and HdaA derepress the AfpyroA transgene, while VeA1 and HstA have no effect on AfpyroA repression. The single mutants (veA1, $\Delta$ hepA, $\Delta c / r D$, $\Delta h s t A$ and $\Delta h d a A$ ) show no effects on radial growth in this assay. (c) Ascosporogenesis assays match the radial growth assays, providing further evidence for the involvement of $\mathrm{HepA}, \mathrm{ClrD}$ and $\mathrm{HdaA}$ in TPE. The single mutants (veA1, $\Delta h e p A, \Delta c / r D, \Delta h s t A$ and $\Delta h d a A$ ) show no effect on ascospore production in this assay. (d) Ascospore production is partially rescued in a double mutant $(\triangle \mathrm{AN5091} \Delta n k u A)$ compared to the single $\triangle \mathrm{AN5091}$ mutant; however, ascospore production does not reach wild-type levels. WT $\left(v e A^{+}\right)$, RDIT9.32; $\triangle$ AN5092::AfpyroA, RJMP121.7; $\triangle$ AN5092::AfpyroA veA1, RJMP121.4; $\Delta$ AN5092:: AfpyroA $\Delta h e p A$, RJMP122.6; $\triangle$ AN5092: : AfpyroA $\Delta c l r D$, RJMP125.20; $\triangle$ AN5092 : : AfpyroA $\Delta h s t A$, RJMP127.4; $\Delta$ AN5092:: AfpyroA $\Delta h d a A$, RJMP123.3; veA1, RDIT2.3; $\Delta h e p A$, RJW110.4; $\Delta c / r D$, RJMP135.11; $\Delta h s t A$, RJMP131.7; $\Delta h d a A$, RMS1.22; $\Delta n k u A$, TJMP45.2; $\Delta$ AN5091, RJMP115.3; and $\Delta$ AN5091 $\Delta n k u A$, TJMP16.1. Note that the $y$-axis scale is logarithmic in panels (c) and (d). Means \pm SD are plotted in panels (b-d); asterisks indicate statistically significant differences between wild-type and other strains at $P<0.001$ using Student's $t$-test.

to wild-type levels, thus indicating that TPE is polygenic in A. nidulans.

\section{DISCUSSION}

TPE is a widespread phenomenon amongst diverse organisms and this work supports the conservation of this silencing mechanism in A. nidulans. Our experiments indicate that transgenes are silenced when placed at the telomere of chromosome III and that this silencing extends at least $30 \mathrm{~kb}$ from the telomere cap to the SpoC1 cluster. We have also identified TPE at the telomere of the right arm of chromosome VI. Additionally, we have shown that
HepA, ClrD, HdaA and NkuA are involved in regulation of TPE at chromosome III.

Our unexpected observation of impaired ascospore development and a reduction in radial growth due to partial repression of $\operatorname{AfpyrG}$ and AfpyroA transgenes led us to exploit these phenotypes to assess TPE at two different telomeres as well as establish a role for NkuA, HepA, ClrD and HdaA in regulating TPE. These simple phenotypic assays are advantageous because they potentially provide a quantitative measure of transgene repression. Sexual development is a complex process in A. nidulans, requiring proper formation of several differentiated cell types that make up a cleistothecium (reviewed by Braus et al., 2002). 
Normal development of the sexual cycle requires adequate nutrient supply, as illustrated by amino acid starvation repression of sexual development (Eckert et al., 1999; Hoffmann et al., 2000), and Bruggeman et al. (2004) reported that many auxotrophic strains are unable to complete the sexual cycle. Extending this observation, the repressed sexual development observed in this study was likely a consequence of inadequate pyridoxine metabolism (pyroA transgene) or pyrimidine metabolism (pyrG transgene); this was substantiated by the finding that growing a pyroA4 mutant on a limited amount of pyridoxine results in loss of ascospore production and eventually a reduction in radial growth (Supplementary Fig. S3 and data not shown).

The KU70/KU80 heterodimer has been shown to be required for non-homologous end joining recombination and normal maintenance of telomeres (Boulton \& Jackson, 1998). The KU heterodimer binds telomeres and is thought to facilitate telomerase activity (reviewed by Dubrana et al., 2001). In the absence of either of the KU proteins, normal telomere function is altered, resulting in lack of heterochromatin complexes and subsequently increased transcription of genes located near telomeres. Similarly to what has been demonstrated in other organisms (Boulton \& Jackson, 1998; Mishra \& Shore, 1999; Rosas-Hernández et al., 2008), we report here that the A. nidulans KU70 homologue (NkuA) partially suppresses TPE as evidenced by increased ascosporogenesis, while at the same time showing that NkuA has no effect on normal ascosporogenesis.

Based on TPE models proposed in other eukaryotic systems, we characterized the involvement of several heterochromatin proteins in TPE of $A$. nidulans. In fission yeast and $N$. crassa, methylation of lysine 9 of histone 3 , heterochromatin protein 1 and histone deacetylase enzymes are involved in regulation of TPE (Ottaviani et al., 2008; Smith et al., 2008). Our results in A. nidulans indicate that there is considerable mechanistic conservation of TPE between fungal species, including TPE regulation by core heterochromatin-activating/maintenance proteins (HepA, ClrD and $\mathrm{HdaA})$. However, there are some differences as well. For example, here we see that NkuA plays a role in A. nidulans TPE, unlike fission yeast, where the KU70 homologue is not involved in TPE. Additionally, Smith et al. (2008) reported that multiple sirtuins (class III histone deacetylases) were involved in regulation of TPE in $N$. crassa, yet we were unable to establish that the $A$. nidulans SIR2 orthologue (HstA) is involved in TPE at chromosome III. Our data suggest involvement of several trans-acting factors in A. nidulans TPE including a Nku complex and histone-remodelling associates.

Chromatin structure has been shown to play a role in transcriptional regulation of genes based on their chromosomal location in several organisms, including $A$. nidulans (Palmer \& Keller, 2010). Here we demonstrate that TPE exists in A. nidulans and show that radial growth and ascosporogenesis can be used as sensitive, quantitative assays to determine the extent of silencing or to identify suppressors of silencing. Future efforts will focus on elucidating any impact of TPE on secondary metabolite gene clusters, which have a tendency to be located in subtelomeric regions (Hoffmeister \& Keller, 2007) and are regulated by some of the same proteins involved in $A$. nidulans TPE (e.g. HdaA, ClrD, HepA) (Reyes-Dominguez et al., 2010; Shwab et al., 2007). Similarly to what has been described in other organisms, we have shown that TPE exists at two telomeres in A. nidulans and predict that TPE likely exists at most telomeres; however, the extent of silencing at an individual telomere may be variable (Mondoux \& Zakian, 2007).

\section{ACKNOWLEDGEMENTS}

This work was funded in part by Gertrude F. Ribble Scholarship to S.M. and D.W.P., and NIH 1 R01 Al065728-01 to N.P.K., PO1GM084077 to B.R.O., C.C.C.W. and N.P.K. and R01GM031837 to B. R. O. The authors thank Marlena Mattingly for construction of TMM11 and Mark Farman for providing information on chromosome III-L telomere structure prior to publication.

\section{REFERENCES}

Adhvaryu, K. K., Morris, S. A., Strahl, B. D. \& Selker, E. U. (2005). Methylation of histone $\mathrm{H} 3$ lysine 36 is required for normal development in Neurospora crassa. Eukaryot Cell 4, 1455-1464.

Arnaud, M. B., Chibucos, M. C., Costanzo, M. C., Crabtree, J., Inglis, D. O., Lotia, A., Orvis, J., Shah, P., Skrzypek, M. S. \& other authors (2010). The Aspergillus Genome Database, a curated comparative genomics resource for gene, protein and sequence information for the Aspergillus research community. Nucleic Acids Res 38, D420D427.

Barrales, R. R., Jimenez, J. \& Ibeas, J. I. (2008). Identification of novel activation mechanisms for FLO11 regulation in Saccharomyces cerevisiae. Genetics 178, 145-156.

Bok, J. W. \& Keller, N. P. (2004). LaeA, a regulator of secondary metabolism in Aspergillus spp. Eukaryot Cell 3, 527-535.

Bok, J. W., Chiang, Y.-M., Szewczyk, E., Reyes-Dominguez, Y., Davidson, A. D., Sanchez, J. F., Lo, H. C., Watanabe, K., Strauss, J. \& other authors (2009). Chromatin-level regulation of biosynthetic gene clusters. Nat Chem Biol 5, 462-464.

Boulton, S. J. \& Jackson, S. P. (1998). Components of the Kudependent non-homologous end-joining pathway are involved in telomeric length maintenance and telomeric silencing. EMBO J 17, 1819-1828.

Braus, G. H., Krappmann, S. \& Eckert, S. E. (2002). Sexual development in ascomycetes fruit body formation of Aspergillus nidulans. In Molecular Biology of Fungal Development, pp. 215-244. Edited by H. Osiewacz. New York: Marcel Dekker, Inc.

Bruggeman, J., Debets, A. J. M. \& Hoekstra, R. F. (2004). Selection arena in Aspergillus nidulans. Fungal Genet Biol 41, 181-188.

Bühler, M. \& Moazed, D. (2007). Transcription and RNAi in heterochromatic gene silencing. Nat Struct Mol Biol 14, 1041-1048.

Castaño, I., Pan, S.-J., Zupancic, M., Hennequin, C., Dujon, B. \& Cormack, B. P. (2005). Telomere length control and transcriptional regulation of subtelomeric adhesins in Candida glabrata. Mol Microbiol 55, 1246-1258. 
Clutterbuck, A. J. \& Farman, M. L. (2008). Aspergillus nidulans linkage map and genome sequence: closing gaps and adding telomeres. In The Aspergilli: Genomics, Medical Aspects, Biotechnology, and Research Methods, pp. 57-73. Edited by G. H. Goldman \& S. A. Osmani. Boca Raton, FL: CRC Press.

Dubrana, K., Perrod, S. \& Gasser, S. M. (2001). Turning telomeres off and on. Curr Opin Cell Biol 13, 281-289.

Eckert, S. E., Hoffmann, B., Wanke, C. \& Braus, G. H. (1999). Sexual development of Aspergillus nidulans in tryptophan auxotrophic strains. Arch Microbiol 172, 157-166.

Freitag, M., Hickey, P. C., Khlafallah, T. K., Read, N. D. \& Selker, E. U. (2004). HP1 is essential for DNA methylation in Neurospora. Mol Cell 13, 427-434.

Gottschling, D. E., Aparicio, O. M., Billington, B. L. \& Zakian, V. A. (1990). Position effect at $S$. cerevisiae telomeres: reversible repression of Pol II transcription. Cell 63, 751-762.

Greenstein, S., Shadkchan, Y., Jadoun, J., Sharon, C., Markovich, S. \& Osherov, N. (2006). Analysis of the Aspergillus nidulans thaumatinlike cetA gene and evidence for transcriptional repression of pyr4 expression in the cetA-disrupted strain. Fungal Genet Biol 43, 42-53.

Grewal, S. I. \& Jia, S. (2007). Heterochromatin revisited. Nat Rev Genet 8, 35-46.

Gwynne, D. I., Miller, B. L., Miller, K. Y. \& Timberlake, W. E. (1984). Structure and regulated expression of the $\mathrm{SpoC1}$ gene cluster from Aspergillus nidulans. J Mol Biol 180, 91-109.

Hoffmann, B., Wanke, C., Lapaglia, S. K. \& Braus, G. H. (2000). c-Jun and RACK1 homologues regulate a control point for sexual development in Aspergillus nidulans. Mol Microbiol 37, 28-41.

Hoffmeister, D. \& Keller, N. P. (2007). Natural products of filamentous fungi: enzymes, genes, and their regulation. Nat Prod Rep 24, 393-416.

Jenuwein, T. \& Allis, C. D. (2001). Translating the histone code. Science 293, 1074-1080.

Laurenson, P. \& Rine, J. (1992). Silencers, silencing, and heritable transcriptional states. Microbiol Rev 56, 543-560.

Lies, C. M., Cheng, J., James, S. W., Morris, N. R., O'Connell, M. J. \& Mirabito, P. M. (1998). BIMAAPC3, a component of the Aspergillus anaphase promoting complex/cyclosome, is required for a G2 checkpoint blocking entry into mitosis in the absence of NIMA function. J Cell Sci 111, 1453-1465.

Louw, C., Young, P. R., van Rensburg, P. \& Divol, B. (2010). Epigenetic regulation of $P G U 1$ transcription in Saccharomyces cerevisiae. FEMS Yeast Res 10, 158-167.

Miller, B. L., Miller, K. Y. \& Timberlake, W. E. (1985). Direct and indirect gene replacements in Aspergillus nidulans. Mol Cell Biol $\mathbf{5}$, 1714-1721.

Mishra, K. \& Shore, D. (1999). Yeast Ku protein plays a direct role in telomeric silencing and counteracts inhibition by rif proteins. Curr Biol 9, 1123-1126.

Mondoux, M. A. \& Zakian, V. A. (2007). Subtelomeric elements influence but do not determine silencing levels at Saccharomyces cerevisiae telomeres. Genetics 177, 2541-2546.

Nayak, T., Szewczyk, E., Oakley, C. E., Osmani, A., Ukil, L., Murray, S. L., Hynes, M. J., Osmani, S. A. \& Oakley, B. R. (2006). A versatile and efficient gene-targeting system for Aspergillus nidulans. Genetics $172,1557-1566$

Ottaviani, A., Gilson, E. \& Magdinier, F. (2008). Telomeric position effect: from the yeast paradigm to human pathologies? Biochimie $\mathbf{9 0}$ 93-107.

Palmer, J. M. \& Keller, N. P. (2010). Secondary metabolism in fungi: does chromosomal location matter? Curr Opin Microbiol 13, 431-436.
Palmer, J. M., Perrin, R. M., Dagenais, T. R. \& Keller, N. P. (2008). H3K9 methylation regulates growth and development in Aspergillus fumigatus. Eukaryot Cell 7, 2052-2060.

Rehmeyer, C., Li, W., Kusaba, M., Kim, Y.-S., Brown, D., Staben, C., Dean, R. \& Farman, M. (2006). Organization of chromosome ends in the rice blast fungus, Magnaporthe oryzae. Nucleic Acids Res 34, 46854701.

Rehmeyer, C. J., Li, W., Kusaba, M. \& Farman, M. L. (2009). The telomere-linked helicase (TLH) gene family in Magnaporthe oryzae: revised gene structure reveals a novel TLH-specific protein motif. Curr Genet 55, 253-262.

Reyes-Dominguez, Y., Bok, J. W., Berger, H., Shwab, E. K., Basheer, A., Gallmetzer, A., Scazzocchio, C., Keller, N. \& Strauss, J. (2010). Heterochromatic marks are associated with the repression of secondary metabolism clusters in Aspergillus nidulans. Mol Microbiol 76, 1376-1386.

Robellet, X., Oestreicher, N., Guitton, A. \& Vélot, C. (2010). Gene silencing of transgenes inserted in the Aspergillus nidulans alcM and/ or alcS loci. Curr Genet 56, 341-348.

Rosas-Hernández, L. L., Juárez-Reyes, A., Arroyo-Helguera, O. E., De Las Peñas, A., Pan, S.-J., Cormack, B. P. \& Castaño, I. (2008). $y K u 70 / y K u 80$ and Rif1 regulate silencing differentially at telomeres in Candida glabrata. Eukaryot Cell 7, 2168-2178.

Schoeftner, S. \& Blasco, M. A. (2009). A 'higher order' of telomere regulation: telomere heterochromatin and telomeric RNAs. EMBO J 28, 2323-2336.

Shaaban, M., Palmer, J., El-Naggar, W. A., El-Sokkary, M. A., Habib, E.-S. E. \& Keller, N. P. (2010). Involvement of transposon-like elements in penicillin gene cluster regulation. Fungal Genet Biol 47, 423-432.

Shimizu, K. \& Keller, N. P. (2001). Genetic involvement of a cAMPdependent protein kinase in a $\mathrm{G}$ protein signaling pathway regulating morphological and chemical transitions in Aspergillus nidulans. Genetics 157, 591-600.

Shwab, E. K., Bok, J. W., Tribus, M., Galehr, J., Graessle, S. \& Keller, N. P. (2007). Histone deacetylase activity regulates chemical diversity in Aspergillus. Eukaryot Cell 6, 1656-1664.

Smith, K. M., Kothe, G. O., Matsen, C. B., Khlafallah, T. K., Adhvaryu, K. K., Hemphill, M., Freitag, M., Motamedi, M. R. \& Selker, E. U. (2008). The fungus Neurospora crassa displays telomeric silencing mediated by multiple sirtuins and by methylation of histone $\mathrm{H} 3$ lysine 9. Epigenetics Chromatin 1, 5.

Szewczyk, E., Nayak, T., Oakley, C. E., Edgerton, H., Xiong, Y., Taheri-Talesh, N., Osmani, S. A. \& Oakley, B. R. (2006). Fusion PCR and gene targeting in Aspergillus nidulans. Nat Protoc 1, 3111-3120.

Tamaru, H. \& Selker, E. U. (2001). A histone H3 methyltransferase controls DNA methylation in Neurospora crassa. Nature 414, 277-283.

Tamaru, H., Zhang, X., McMillen, D., Singh, P. B., Nakayama, J., Grewal, S. I., Allis, C. D., Cheng, X. \& Selker, E. U. (2003). Trimethylated lysine 9 of histone H3 is a mark for DNA methylation in Neurospora crassa. Nat Genet 34, 75-79.

Timberlake, W. E. (1980). Developmental gene regulation in Aspergillus nidulans. Dev Biol 78, 497-510.

Tsitsigiannis, D. I., Zarnowski, R. \& Keller, N. P. (2004). The lipid body protein, PpoA, coordinates sexual and asexual sporulation in Aspergillus nidulans. J Biol Chem 279, 11344-11353.

Yang, L., Ukil, L., Osmani, A., Nahm, F., Davies, J., De Souza, C. P., Dou, X., Perez-Balaguer, A. \& Osmani, S. A. (2004). Rapid production of gene replacement constructs and generation of a green fluorescent protein-tagged centromeric marker in Aspergillus nidulans. Eukaryot Cell 3, 1359-1362.

Edited by: S. D. Harris 